\title{
Cytochrome P450 2E1 variable number tandem repeat polymorphisms and health risks: A genotype-phenotype study in cancers associated with drinking and/or smoking
}

\author{
IRENE CATANZARO $^{1}$, FLORES NASELLI ${ }^{1}$, MARGHERETH SAVERINI ${ }^{1}$, ANTONIO GIACALONE ${ }^{2}$, \\ GIUSEPPE MONTALTO $^{2}$ and FABIO CARADONNA ${ }^{1}$
}

\author{
${ }^{1}$ Department of Molecular and Biomolecular Sciences and Technologies (STEMBIO), Section of Cellular Biology, \\ University of Palermo, I-90128 Palermo; ${ }^{2}$ Department of Internal and Specialist Medicine, \\ University of Palermo, I-90100 Palermo, Italy
}

Received January 31, 2012; Accepted April 25, 2012

DOI: $10.3892 / \mathrm{mmr} .2012 .914$

\begin{abstract}
Cytochrome P450 2E1 (CYP2E1) is one of the main enzymes involved in the oxidation of ethanol and in the transformation of a number of potentially dangerous compounds. It has various polymorphic sites, one of which is a variable number tandem repeat (VNTR) polymorphism previously described in the 5'-flanking region. The aim of this study was to investigate the genotype-phenotype association between CYP2E1 VNTR polymorphisms and risky health habits in healthy subjects and to analyze the associations between these polymorphisms with drinking- and/or smoking-related cancers. We analyzed 166 healthy subjects by genotyping for the CYP2E1 VNTR polymorphism associated with drinking and/or smoking habits by the more sensitive restriction fragment length polymorphism-polymerase chain reaction (RFLP-PCR) method, using the NlaIV restriction enzyme. Sixty cases of pancreatic adenocarcinoma (PA) and 66 with hepatocellular carcinoma (HCC), were also genotyped. Statistical analysis was carried out to investigate the genotype-phenotype associations and to compare certain genotypes and cancer. We found 7 genotypes both in the healthy subjects and patients. The A1/A1 genotype was observed to be mainly associated with non-drinkers and -smokers (87.5 and $75.0 \%$, respectively); moreover it was never found in the PA or HCC patients. Conversely, a weak association between A2/A3 with smokers (45.8\%) and A4/A4 with drinkers (53.9\%) was detected. In addition, the A4/A4 genotype was found to be significantly associated to PA [odds ratio $(\mathrm{OR})=3.25 ; 95 \%$ confidence interval $(\mathrm{CI}) 1.21-7.50]$. Our
\end{abstract}

Correspondence to: Professor Fabio Caradonna, Department of Molecular and Biomolecular Sciences and Technologies (STEMBIO), Section of Cellular Biology, University of Palermo, Viale delle Scienze, Edificio 16, I-90128 Palermo, Italy

E-mail: fabio.caradonna@unipa.it

Key words: human genetic variability, genetic factors, cytochrome P450 2E1 variable number tandem repeat polymorphisms, predisposing alleles, health risks, drinking- and/or smoking-related cancer data demonstrate that certain CYP2E1 VNTR genotypes are associated with drinking and/or smoking habits; consequently, they may contribute either to the decreased or increased risk of developing drinking- and/or smoking-related cancers. In particular, we hypothesize that the A1/A1 VNTR genotype may have a protective role against drinking- and/or smokingrelated cancers, and that A4/A4 may be a high-risk genotype during the early stages of cancer.

\section{Introduction}

During the last few years, it has been documented that alcoholism is increasing among young persons (1). Alcohol is metabolized by the liver cells (hepatocytes) via 3 pathways: the alcohol dehydrogenase pathway in the cytosol, the microsomal ethanol oxidizing system in the endoplasmic reticulum and catalase in the peroxisomes. All 3 pathways result in the production of acetaldehyde, which is then metabolized by aldehyde dehydrogenase (ALDH) into acetate, a metabolite more toxic than alcohol itself (2-4).

Cytochrome P450 2E1 (CYP2E1) is the main enzyme in the microsomal ethanol oxidation system (5), and it is a member of the cytochrome P450 (CYP) superfamily, a membrane-bound protein primarily associated with endoplasmic reticulum membranes. CYP2E1 has also been shown to be involved in the metabolism of more than 80 low-molecular hydrophobic toxicologically dangerous compounds and contributes to the activation of a number of pro-carcinogens and drugs into highly reactive metabolites (6,7). Specifically, CYP2E1 activates $\mathrm{N}$-nitrosamines contained in tobacco smoke and foodstuffs (8-10) and several industrial (11) and endogenous carcinogens $(12,13)$; moreover, it is capable of reducing molecular oxygen to highly reactive compounds, such as superoxide anion radical $\left(\mathrm{O}_{2}{ }^{-}\right)$, singlet oxygen $\left({ }^{1} \mathrm{O}_{2}\right)$, hydrogen peroxide $\left(\mathrm{H}_{2} \mathrm{O}_{2}\right)$ and hydroxyl radical $(\mathrm{OH})$ leading to DNA damage and carcinogenesis $(14,15)$.

The human CYP2E1 gene is located on chromosome 10q26.3 and consists of 9 exons and 8 introns. CYP2E1 gene expression may be regulated at the transcription and translation level, as well as the mRNA stabilization and protein degrada- 
tion levels $(16,17)$. Similar to other CYP genes, CYP2E1 has more than 10 different polymorphic sites, in the 5'-flanking region, in introns and transcribed gene regions. In particular, a polymorphic variable number tandem repeat (VNTR) sequence has been described $\sim 2.0 \mathrm{~kb}$ pair upstream of the transcription start site (18).

There is a high variability among different ethnic groups in the activity of the CYP enzyme due to both environmental and genetic factors, such as polymorphisms. Polymorphic CYP genes are capable of creating differences in the ability to metabolize, detoxify or activate a number of substances. This ability, as is well known, provides one of the first lines of defence against xenobiotic chemicals; therefore, we hypthesized that an alteration in the activity of these enzymes may result in a greater susceptibility to certain diseases or cancer, although existing evidence on the matter is controversial (19). A number of studies have clarified that certain polymorphic genes, in association with alcohol consumption, are involved in the development of certain cancers (20-22). Despite the fact that alcohol is not itself a carcinogen, it acts as a co-carcinogen by enhancing the effects of other chemicals activated by certain enzymes, i.e. CYP2E1. Certain studies have demonstrated that CYP2E1 is highly expressed in both the liver and pancreas after ethanol administration (23). Acetaldehyde produced during ethanol oxidation may be the direct cause of cell damage via the production of reactive oxygen species (ROS). Moreover, it is currently well known that certain tumours are heritable $(24,25)$ and that environmental hazards associated with particular habits may increase the risk of tumour insurgence. In this study, we investigated CYP2E1 VNTR polymorphism distribution among healthy subjects by analyzing whether particular CYP2E1 VNTR polymorphisms were associated with certain health-risk behaviours, such as alcohol consumption and/or cigarette smoking. Finally we analyzed patients with pancreatic adenocarcinoma (PA) and hepatocellular carcinoma (HCC) in order to find evidence of a possible correlation between certain CYP2E1 VNTR polymorphisms, risky habits and predisposition to cancer.

\section{Subjects and methods}

Healthy subjects and patients. In this study, 166 apparently healthy subjects (106 females and 60 males, aged 19 to 34 years, residents of sourthern Italy) were enrolled. All the patients and subjects gave their written informed consent to participate in the study. A questionnaire to determine their age, as well as their drinking and smoking habits was compiled by each person. Accordingly, the subjects were considered as drinkers if they drank approximately 1 glass/day and as smokers if they smoked between 1-5 cigarettes/day. Moreover, to better understand the possible association between CYP2E1 VNTR genotypes and drinking- and/or smoking-related neoplastic diseases, we genotyped 60 cases of PA and 66 with HCC, collected from the same geographical area.

DNA extraction. Genomic DNA was obtained from the saliva of healthy subjects and from the peripheral blood lymphocytes of patients. DNA was purified using a PureLink ${ }^{\mathrm{TM}}$ Genomic DNA mini kit according to the manufacturer's instructions (Invitrogen).
Table I. Summary of the phenotype study on healthy subjects.

\begin{tabular}{lr}
\hline Variable & $\begin{array}{r}\text { Subjects } \\
\text { no. }(\%)\end{array}$ \\
\hline Gender & $166(100)$ \\
Male & $60(36.1)$ \\
Female & $106(63.9)$ \\
Age (mean \pm SD) & $21.2 \pm 2.2$ \\
Male & $21.8 \pm 3.0$ \\
Female & $21.3 \pm 2.4$ \\
Drinking habit & \\
Never & $117(70.5)$ \\
Ever & $49(29.5)$ \\
Smoking habit & \\
Never & $113(68.1)$ \\
Ever & $53(31.9)$ \\
\hline
\end{tabular}

Genotyping. To genotype the CYP2E1 VNTR polymorphism, we performed restriction fragment length polymorphismpolymerase chain reaction (RFLP-PCR) using a final volume of $50 \mu \mathrm{l}$ containing $150 \mathrm{ng}$ of genomic DNA, $0.2 \mathrm{mM}$ of each dNTP, $1.5 \mathrm{mM}$ of $\mathrm{MgCl}_{2}, 0.05 \mathrm{U} / \mu \mathrm{l}$ of Taq polymerase (Invitrogen) and $0.4 \mu \mathrm{M}$ of each primer: 5'-CAC ACC CAG CCA ACA GCA G-3' (sense) and 5'-TGC ATC TGT CCC ATT GGC AG-3' (antisense) (16). PCR was carried out by 30 thermal cycles under the following conditions: $4 \mathrm{~min}$ at $94^{\circ} \mathrm{C}$ for initial melting, $1 \mathrm{~min}$ at $94^{\circ} \mathrm{C}$ for melting, $1 \mathrm{~min}$ at $58^{\circ} \mathrm{C}$ for primer annealing and $1 \mathrm{~min}$ at $72^{\circ} \mathrm{C}$ for primer extension.

Half of the PCR product was subjected to restriction endonuclease digestion by NlaIV $(0.5 \mathrm{U} / \mu 1$, BioLabs $)$ at $37^{\circ} \mathrm{C}$ for $2 \mathrm{~h}$. Digested and non-digested samples were analyzed by a $12 \%$ polycrylamide gel stained with SYBR Safe DNA gel stain (Invitrogen) and visualized by ChemiDoc XRS (Bio-Rad).

Statistical analysis. Statgraphics data analysis (plus version 5.1) was used to investigate the associations between genotypes and drinking and/or smoking. In order to evaluate the heredity equilibrium, we used the Hardy-Weinberg test. The association of the genotypes with the risk of PA or HCC was investigated by evaluating the odds ratio (OR) and 95\% confidence interval (CI) using SPSS software.

\section{Results}

Phenotype distribution. Table I shows some information obtained from the phenotype study carried out on healthy subjects. As can be observed, the population consisted of $63.9 \%$ females and $36.1 \%$ males. No significant difference between the genders was observed regarding the mean age (21.8 \pm 3.0 vs. $21.3 \pm 2.4$ years, for males and females, respectively).

Genotype distribution. Table II shows the CYP2E1 VNTR genotype frequencies detected in the healthy subjects. We found 7 VNTR-CYP2E1 genotypes: A1/A1, A1/A2, A2/A2, A2/A3, A2/A4, A3/A 3 and A4/A4. The most frequent genotype was A2/A2 (63.8\%), and A1/A2 and A3/A3 were the least 
Table II. CYP2E1 VNTR genotype distribution in healthy subjects.

\begin{tabular}{lccccccccccc}
\hline & \multicolumn{1}{c}{ CYP2E1 VNTR genotype, no. (\%) } \\
\cline { 2 - 9 } & A1/A1 & A1/A2 & A1/A3 & A1/A4 & A2/A2 & A2/A3 & A2/A4 & A3/A3 & A3/A4 & A4/A4 & no. (\%) \\
\hline $\begin{array}{l}\text { Healthy } \\
\text { subjects }\end{array}$ & $16(9.6)$ & $1(0.6)$ & 0 & 0 & $106(63.8)$ & $24(14.5)$ & $5(3.0)$ & $1(0.6)$ & 0 & $13(7.8)$ & $166(100)$
\end{tabular}

CYP2E1, cytochrome P450 2E1; VNTR, variable number tandem repeat.

Table III. Distribution of CYP2E1 genotypes according to alcohol consumption in healthy subjects.

\begin{tabular}{|c|c|c|c|c|c|c|c|c|c|c|c|}
\hline \multirow{2}{*}{$\begin{array}{l}\text { Healthy } \\
\text { subjects }\end{array}$} & \multicolumn{10}{|c|}{ CYP2E1 VNTR genotype, no. (\%) } & \multirow{2}{*}{$\begin{array}{c}\text { Total } \\
\text { no. }(\%)\end{array}$} \\
\hline & A1/A1 & $\mathrm{A} 1 / \mathrm{A} 2$ & A1/A3 & A1/A4 & $\mathrm{A} 2 / \mathrm{A} 2$ & A2/A3 & A2/A4 & A3/A3 & A3/A4 & A4/A4 & \\
\hline Non-drinkers & $14(87.5)$ & $1(100)$ & 0 & 0 & $73(68.9)$ & $18(75.0)$ & $4(80.0)$ & $1(100)$ & 0 & $6(46.1)$ & $117(70.5)$ \\
\hline Drinkers & $2(12.5)$ & 0 & 0 & 0 & $33(31.1)$ & $6(25.0)$ & $1(20.0)$ & 0 & 0 & $7(53.9)$ & $49(29.5)$ \\
\hline $\mathrm{OR}^{\mathrm{a}}$ & 0.31 & 1.00 & & & 1.36 & 0.76 & 0.58 & 1.00 & & 3.08 & \\
\hline $95 \% \mathrm{CI}$ & $0.06-1.43$ & $0.99-1.02$ & & & $0.66-2.78$ & $0.28-2.06$ & $0.06-5.40$ & $0.99-1.02$ & & $0.97-9.70$ & \\
\hline
\end{tabular}

CYP2E1, cytochrome P450 2E1; VNTR, variable number tandem repeat. ${ }^{a} \mathrm{OR}$, odds ratio (calculated vs. non-drinkers); CI, confidence interval.

Table IV. Distribution of CYP2E1 genotypes according to smoking habit in healthy subjects.

\begin{tabular}{|c|c|c|c|c|c|c|c|c|c|c|c|}
\hline \multirow{2}{*}{$\begin{array}{l}\text { Healthy } \\
\text { subjects }\end{array}$} & \multicolumn{10}{|c|}{ CYP2E1 VNTR genotype, no. (\%) } & \multirow{2}{*}{$\begin{array}{c}\text { Total } \\
\text { no. }(\%)\end{array}$} \\
\hline & A1/A1 & $\mathrm{A} 1 / \mathrm{A} 2$ & A1/A3 & A1/A4 & $\mathrm{A} 2 / \mathrm{A} 2$ & A2/A3 & $\mathrm{A} 2 / \mathrm{A} 4$ & A3/A3 & A3/A4 & $\mathrm{A} 4 / \mathrm{A} 4$ & \\
\hline Non-smokers & $12(75.0)$ & $1(100)$ & 0 & 0 & $73(69.8)$ & $12(50.0)$ & $5(100)$ & $1(100)$ & 0 & $9(69.2)$ & $113(68.1)$ \\
\hline Smokers & $4(25.0)$ & 0 & 0 & 0 & $33(30.2)$ & $12(50.0)$ & 0 & 0 & 0 & $4(30.8)$ & $53(31.9)$ \\
\hline $\mathrm{OR}^{\mathrm{a}}$ & 0.68 & 1.00 & & & 1.06 & 2.73 & 1.04 & 1.00 & & 0.94 & \\
\hline $95 \% \mathrm{CI}$ & $0.21-2.24$ & $0.99-1.02$ & & & $0.53-2.11$ & $1.15-6.50$ & $1.00-1.08$ & 0.99-1.02 & & $0.27-3.21$ & \\
\hline
\end{tabular}

CYP2E1, cytochrome P450 2E1; VNTR, variable number tandem repeat. ${ }^{a} \mathrm{OR}$, odds ratio (calculated vs. non-smokers); CI, confidence interval.

frequent $(0.6 \%)$, whereas genotypes A1/A3, A1/A4 and A3/A4 were not detected. All the genotypes detected in this study were in Hardy Weinberg equilibrium.

Genotype association with habitual drinking. In order to investigate whether particular CYP2E1 VNTR polymorphisms are associated with alcohol consumption, we differentiated the frequencies of non-drinkers or drinkers within each genotype; the results are reported in Table III. As can be observed, the A4/A4 genotype increased the OR for drinking habit $(3.08 ; 95 \%$ CI 0.97-9.70) while the A1/ A1 genotype was not associated with any increased OR for drinking habit.

Genotype association with habitual smoking. In order to investigate whether particular CYP2E1 VNTR polymorphisms are associated with habitual smoking, we differentiated the frequencies of non-smokers or smokers within each genotype; the results are reported in Table IV. The A2/A3 genotype for smoking habit increased the OR $(2.73$; 95\% CI 1.15-6.50) and interestingly, the A1/A1 genotype was not associated with any increased OR for this risky habit.

Genotype association with healthy subjects and cancer cases. The results presented in Table $\mathrm{V}$ show the distribution of CYP2E1 genotypes in healthy subjects, PA and HCC patients. It is worth noting that no cases, neither PA nor HCC with the A1/A1 genotype were found. Moreover a significant difference was found in the correlation with PA; in fact there was a greater percentage of PA cases with the A4/A4 genotype $(\mathrm{OR}=3.25 ; 95 \%$ CI 1.41-7.50). No significant differences were detected in the HCC cases. 
Table V. Distribution of CYP2E1 genotypes in healthy subjects, PA and HCC patients.

\begin{tabular}{|c|c|c|c|c|c|c|c|c|c|c|c|}
\hline & \multicolumn{10}{|c|}{ CYP2E1 VNTR genotype, no. (\%) } & \multirow{2}{*}{$\begin{array}{c}\text { Total } \\
\text { no. }(\%)\end{array}$} \\
\hline & $\mathrm{A} 1 / \mathrm{A} 1$ & $\mathrm{~A} 1 / \mathrm{A} 2$ & A1/A3 & A1/A4 & A2/A2 & A2/A3 & $\mathrm{A} 2 / \mathrm{A} 4$ & $\mathrm{~A} 3 / \mathrm{A} 3$ & A3/A4 & $\mathrm{A} 4 / \mathrm{A} 4$ & \\
\hline Control & $16(9.64)$ & $1(0.60)$ & 0 & 0 & $106(63.86)$ & $24(14.46)$ & $5(3.01)$ & $1(0.60)$ & 0 & $13(7.83)$ & $166(100)$ \\
\hline PA & 0 & 0 & 0 & 0 & $38(63.33)$ & $4(6.67)$ & $5(8.33)$ & 0 & 0 & $13(21.67)$ & $60(100)$ \\
\hline $\mathrm{OR}^{\mathrm{a}}$ & 1.10 & 1.00 & & & 0.97 & 0.42 & 2.97 & 1.00 & & 3.25 & \\
\hline $95 \% \mathrm{CI}$ & $1.05-1.16$ & $0.99-1.01$ & & & $0.53-1.80$ & $0.14-1.27$ & $0.81-10.49$ & $0.99-1.01$ & & $1.41-7.50$ & \\
\hline $\mathrm{HCC}$ & 0 & $2(3.03)$ & 0 & 0 & $52(78.79)$ & $11(16.67)$ & 0 & 0 & 0 & $1(1.51)$ & $66(100)$ \\
\hline $\mathrm{OR}^{\mathrm{a}}$ & 1.10 & 5.15 & & & 2.12 & 1.18 & 1.03 & 1.00 & & 0.18 & \\
\hline $95 \% \mathrm{CI}$ & $1.05-1.16$ & $0.46-57.8$ & & & $1.07-4.10$ & $0.54-2.57$ & $1.00-1.05$ & $0.99-1.01$ & & $0.02-1.41$ & \\
\hline
\end{tabular}

CYP2E1, cytochrome P450 2E1; VNTR, variable number tandem repeat. PA, pancreatic adenocarcinoma; HCC, hepatocellular carcinoma; ${ }^{a} \mathrm{OR}$, odds ratio (calculated vs. control); CI, confidence interval.

\section{Discussion}

It is known that alcohol consumption and cigarette smoking are, unfortunately, increasing among young persons (1). Moreover, it is well known that these dangerous injuries exert an influence on susceptibility to tumours, such as PA (26) and HCC. These social and clinical problems drove us to explore whether an association exists between particular genetic factors, health-risk behaviours, as well as drinking- and/or smokingrelated neoplastic diseases. We focused our attention on CYP genes and in particular, on CYP2E1, which is a polymorphic gene involved not only in the ethanol oxidative metabolism, but also in the transformation of many exogenous compounds, including N-nitrosamines, which may be activated into highly reactive metabolites. Polymorphisms in this gene can create differences in the ability to metabolize, detoxify or activate a number of substances. This ability can generate certain reactive substances which may consequently increase the risk of developing serious diseases, such as tumours $(7,8,14,27)$.

The VNTR polymorphism in the 5'-flanking region of the CYP2E1 gene has previously been described (18). Moreover, to date, only a few studies have focused on this polymorphism and its asociation with drinking and/or smoking and cancer development (16). We formed the hypothesis that the VNTR polymorphism may be responsible (together with others) for the different susceptibility to serious diseases which are caused, directly or indirectly, by drinking and/or smoking. In this study, we report for the first time, the results of VNTR CYP2E1 genotyping, carried out in 166 young healthy individuals and 126 patients (60 PA and $66 \mathrm{HCC}$ ), residents of southern Italy.

In order to genotype the VNTR polymorphisms, we developed a simple and precise technique in our laboratory, suitable as a hospital diagnostic routine, consisting of PCR followed by enzyme digestion to improve sensitivity and resolution.

We found 7 out of 10 expected genotypes: A2/A2 was the most frequent and A1/A2, A3/A3 were the least frequent both in the healthy subjects and patients. The genotypes, A1/A3, A1/A4 and A3/A4, were not detected. As A2/A2 was the modal genotype in all the studied subjects, we believe that this allele provides a generic selective advantage in humans.
We considered the results regarding the other no-modal genotypes more interesting. In fact, in all the association analyses between the health-risk behaviours and VNTR genotypes, we found that individuals with the A1/A1 genotype had a lower inclination either to drink or smoke (the lowest OR values shown in Tables III and IV), whereas individuals with the A4/A4 genotype had an inclination to drink and those with the A2/A3 genotype a propensity to smoke. Consequently, individuals with the A1/A1 genotype are exposed neither to potentially dangerous molecules inhaled by smoking and transformed by the CYP2E1 enzymes, nor to the ROS produced by the CYP over-activity induced by alcohol ingestion. This genotype may determine a lower susceptibility to drinking- and/or smokingrelated neoplastic diseases, hence it may be considered a protective genetic order. Otherwise, A4/A4 subjects are exposed to the continual alcohol induction of the CYP2E1 gene; therefore, they are promptly able to transfrom certain incidentally absorbed pro-carcinogens into active toxic molecules and to increase CYP activity-derived ROS production. This genotype, therefore, may be associated with the susceptibility to develop serious drinking-related diseases.

These results led us to investigate whether an association between the A4/A4 genotype and tumour susceptibility exists; therefore, we genotyped patients with drinking- and/or smoking-related tumours (i.e. PA and HCC). In confirmation of the results obtained from the healthy subjects, the A1/A1 genotype was found neither among PA nor HCC patients and A4/A4 PA cases were significant ( 3 -fold $)$ with respect to the controls (OR=3.25; 95\% CI 1.21-7.50) (Table V).

Nowadays, little is known about the risk factors for pancreatic cancer (20) and in particular, there are controversial data about alcohol consumption (28) as a susceptibility factor. Our results show that one of the VNTR CYP2E1 genotypes could be a susceptibility factor for PA, either directly, by conferring a higher expression of the CYP2E1 gene, or indirectly, by being associated with drinking habits. It has previously been reported that transcriptional activity is increased in subjects with the A4 allele (16) and the inductive effect of ethanol on CYP2E1 gene expression (12) and on ROS production is well known (29). 
We hypothesized that the A4/A4 genotype, having a double dose of the A4 allele, may be associated with a greater susceptibility to PA, confirming the suspicions reported previously regarding other cancers (16), especially for subjects which have an inclination to alcohol consumption.

We consider that further studies are required to further confirm this hypothesis. In particular, it may be of interest to study the real contribution of VNTR alleles in CYP2E1 gene expression, as well as their regulatory elements contained in the 5 '-flanking region, either genetic, or epigenetic.

\section{Acknowledgements}

The authors are grateful to Professor G. Barbata and Professor G. Sciandrello for their constructive discussion. Moreover, they would like to thank Professor L. Marasà for his scientific collaboration, and ASBIP (Associazione Scientifica Biologi Palermo) for its helpful financial support. This study was mainly supported by Fondo di Ateneo 2007 (Università di Palermo; F.C.).

\section{References}

1. Hemmingsson T and Lundberg I: Work control, work demands, and work social support in relation to alcoholism among young men. Alcohol Clin Exp Res 22: 921-927, 1998.

2. Goedde HW and Agarwal DP (eds): Acetaldehyde metabolism: genetic variation and physiological implications. In: Alcoholism: Biomedical and Genetic Aspects. Pergamon Press, Elmsford, NY, pp21-56, 1989.

3. Bosron WF, Ehrig T and Li TK: Genetic factors in alcohol metabolism and alcoholism. Semin Liver Dis 13: 126-135, 1993.

4. Lieber CS: Alcohol and the liver: 1994 update. Gastroenterology 106: 1085-1105, 1994

5. Lewis DF, Bird MG and Parke DV: Molecular modelling of CYP2E1 enzymes from rat mouse and man: an explanation for species differences in butadiene metabolism and potential carcinogenicity, and rationalization of CYP2E substrate specificity. Toxicology 118: 93-113, 1997.

6. Umeno M, McBride OW, Yang CS, Gelboin HV and Gonzalez FJ: Human ethanol-inducible P450IIE1: complete gene sequence, promoter characterization, chromosome mapping and cDNAdirected expression. Biochemistry 27: 9006-9013, 1988.

7. Guengerich FP, Kim DH and Iwasaki M: Role of human cytochrome P450IIE1 in the oxidation of many low molecular weight cancer suspects. Chem Res Toxicol 4: 168-179, 1991.

8. Nakajima T and Aoyama T: Polymorphism of drug-metabolizing enzymes in relation to individual susceptibility to industrial chemicals. Ind Health 38: 143-152, 2000.

9. Wang AH, Sun CS, Li LS, Huang JY and Chen QS: Relationship of tobacco smoking CYP1A1 GSTM1 gene polymorphism and esophageal cancer in Xi'an. World J Gastroenterol 8: 49-53, 2002.

10. Bartsch H, Nair U, Risch A, Rojas M, Wikman H and Alexandrov K: Genetic polymorphism of CYP genes, alone or in combination, as a risk modifier of tobacco-related cancers Cancer Epidemiol Biomarkers Prev 9: 3-28, 2000.
11. Ingelman-Sundberg M, Johansson I, Yin H, Terelius $\mathrm{Y}$, Eliasson E, Clot P and Albano E: Ethanol-inducible cytochrome P4502E1: genetic polymorphism, regulation, and possible role in the etiology of alcohol-induced liver disease. Alcohol 10: 447-452, 1993 .

12. Hu Y, Oscarson M, Johansson I, et al: Genetic polymorphism of human CYP2E1: characterization of two variant alleles. Mol Pharmacol 51: 370-376, 1997.

13. Agundez JA: Cytochrome P450 gene polymorphism and cancer. Curr Drug Metab 5: 211-224, 2004.

14. Koop DR: Oxidative and reductive metabolism by cytochrome P450 2E1. FASEB J 6: 724-730, 1992.

15. Lieber CS: Microsomal ethanol oxidizing system (MEOS): the first 30 years (1968-1998) - a review. Alcohol Clin Exp Res 23: 991-1007, 1999.

16. Itoga S, Nomura F, Makino Y, et al: Tandem repeat polymorphism of the CYP2E1 gene: an association study with esophageal cancer and lung cancer. Alcohol Clin Exp Res 26: 15S-19S, 2002.

17. Danko IM and Chaschin NA: Association of CYP2E1 gene polymorphism with predisposition to cancer development. Exp Oncol 27: 248-256, 2005.

18. Hayashi S, Watanabe J and Kawajiri K: Genetic polymorphisms in the 5'-flanking region change transcriptional regulation of the human cytochrome P450IIE1 gene. J Biochem 110: 559-565, 1991.

19. Watanabe M: Polymorphic CYP genes and disease predisposition - what have the studies shown so far? Toxicol Lett 102-103. 167-171, 1998.

20. Yang B, O'Reilly DA, Demaine AG and Kingsnorth AN: Study of polymorphisms in the CYP2E1 gene in patients with alcoholic pancreatitis. Alcohol 23: 91-97, 2001.

21. Yu SZ, Huang XE, Koide T, et al: Hepatitis B and C viruses infection, lifestyle and genetic polymorphisms as risk factors for hepatocellular carcinoma in Haimen, China. Jpn J Cancer Res 93: 1287-1292, 2002.

22. Munaka M, Kohshi K, Kawamoto T, et al: Genetic polymorphisms of tobacco- and alcohol-related metabolizing enzymes and the risk of hepatocellular carcinoma. J Cancer Res Clin Oncol 129: 355-360, 2003.

23. Norton ID, Apte MV, Haber PS, McCaughan GW, Pirola RC and Wilson JS: Cytochrome P4502E1 is present in rat pancreas and is induced by chronic ethanol administration. Gut 42: 426-430, 1998.

24. Caporaso NE: The genetics of lung cancer. In: The Genetics of Cancer. Ponder BAJ and Waring MJ (eds). Kluwer Academic, Dordrecht, pp21-43, 1995.

25. Claus EB: The genetic epidemiology of cancer. Cancer Surv 25: 13-26, 1995.

26. Talamini G, Bassi C, Falconi M, et al: Alcohol and smoking as risk factors in chronic pancreatitis and pancreatic cancer. Dig Dis Sci 44: 1303-1311, 1999.

27. Cederbaum AI: Microsomal generation of reactive oxygen species and their possible role in alcohol hepatotoxicity. Alcohol Alcohol Suppl 1: 291-296, 1991.

28. Brand RE, Greer JB, Zolotarevsky E, et al: Pancreatic cancer patients who smoke and drink are diagnosed at younger ages. Clin Gastroenterol Hepatol 7: 1007-1012, 2009.

29. Lu Y and Cederbaum AI: CYP2E1 and oxidative liver injury by alcohol. Free Radic Biol Med 44: 723-738, 2008. 